\title{
The Case for General Collective Intelligence Rather than Artificial General Intelligence being the Most Important Human Innovation in the History and Future of Mankind
}

\author{
Andy E. Williams, Nobeah Foundation, Nairobi, Kenya
}

\begin{abstract}
Artificial General Intelligence, that is an Artificial Intelligence with the ability to redesign itself and other technology on its own, has been called "mankind's last invention", since it may not only remove the necessity of any human invention afterwards, but also might design solutions far too complex for human beings to have the ability to contribute to in any case. Because of this, if and when AGI is ever invented, it has been argued by many that it will be the most important innovation in the history of the mankind up to that point. Just as nature's invention of human intelligence might have transformed the entire planet and generated a greater economic impact than any other innovation in the history of the planet, AGI has been suggested to have the potential for an economic impact larger than that resulting from any other innovation in the history of mankind. This paper explores the case for General Collective Intelligence being a far more important innovation than AGI. General Collective Intelligence has been defined as a solution with the capacity to organize groups of human or artificial intelligences into a single collective intelligence with vastly greater general problem solving ability. A recently proposed model of GCI not only outlines a model for cognition that might also enable AGI, but also identifies hidden patterns in collective outcomes for groups that might make GCI necessary in order to reliably achieve the benefits of AGI while reliably avoiding the potentially catastropic costs of AGI.
\end{abstract}

\section{Keywords}

AGI artificial intelligence artificial general intelligence risk singularity superintelligence safe AI future of AI whole brain emulation intelligence GCI general collective intelligence

\section{Introduction}

Human intelligence has enabled mankind to generate a surplus of resources, but many other animals also generate a surplus of territory, food, or other resources in order to attract a mate, to nurture offspring, or to achieve other purposes. However, human intelligence has also given mankind sufficient capacity for abstraction to enable that surplus to be represented in abstract terms that remove the barriers to accumulation. Whether accumulation of knowledge of where fruits or vegetables can be gathered, whether accumulation of reasoning processes enabling prey animals to be outwitted or predator animals to be escaped, with such abstraction any accumulation can potentially be represented as abstract economic value that can be stored and exchanged. And this removal of the barriers to accumulating economic value has enabled human-beings to accumulate orders of magnitude greater value than any other organism.

If it is true that no other animal has had the intelligence to have been able to abstract value into a form that can be accumulated in this way, then the economic value of human intelligence might be at least the value of all economic wealth that has been accumulated, that is, all the wealth in existence on the earth. While the nature of this wealth, and hence it's value, might be represented partially by intangible capital like the value of the rule of law or the value of a civilization's institutions [1], assuming that human civilizations are not also accumulating environmental or other costs that eventually need to be repaid, the entire Gross World Product (GWP) might provide a rough order of magnitude estimate of this value. In 2017, according to the CIA's World Factbook, the GWP was around US\$80.27 trillion in nominal terms and totaled approximately 127.8 trillion international dollars in terms of purchasing power parity (PPP). No other single innovation but human intelligence can be said to be responsible for all this economic activity. For this reason human intelligence, in terms of economic impact, might be 
nature's most important innovation in the entire 3.5 billion year history of the earth. Random events like mass extinctions due to asteroid strikes might be more impactful in terms of sheer volume of biomass affected. But from the point of view of the terrestrial environment being an adaptive system, such events are external rather than the consequence of internal adaptative innovation.

Human intelligence is not only likely the most impactful innovation in the history of the planet, but it also might be the most impactful innovation possible in the planet's future until a similarly transformative adaptive change might be achieved. A recently developed Functional Modeling Framework (FMF) [19] for modeling cognition and other functional components of living systems defines cognition as navigating a "conceptual space". Defining each path through cognitive space as solving a problem, and assuming each path has a uniform cross-sectional area, the FMF defines the intelligence factor (general problem solving ability) of a cognitive system as being proportional to the number of paths, and therefore to the volume of cognitive space, that can be navigated per unit time.

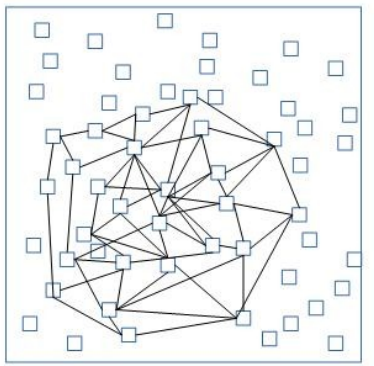

Larger volume of conceptual space navigated per unit time (higher general problem solving ability).

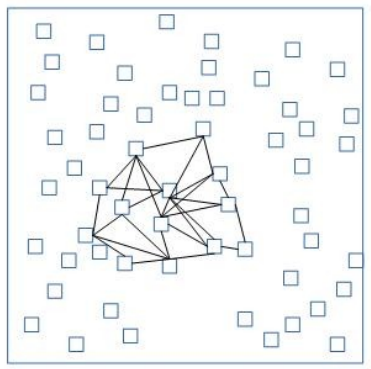

Smaller volume of conceptual space navigated per unit time (lower general problem solving ability).

Figure 1: General problem solving ability.

As mentioned, human intelligence is suggested to be unique in that the problems it can solve enables it to remove the barriers to creating and accumulating orders of magnitude greater value. If so, then the ability of a new cognitive system to navigate a still larger cognitive space, and it's ability to resolve finer detail in cognitive space, might sufficiently increase the density of points that can be navigated to make solving entirely new problems possible, and therefore to facilitate accumulation of new categories of value to make accumulation possible at many orders of magnitude higher still. Such an ability might represent such a transformative change that it replaces human intelligence in importance. Until that point of transformation however, no innovation resulting from human intelligence, whether discovering the wheel, the printing press, electricity, the internal combustion engine, or penicillin, can be more important than nature's invention of intelligence itself.

The FMF defines cognition to be a pattern of dynamical stability in the fitness of a cognitive system to function. In the FMF the cognitive system's ability to adapt to achieve stability of fitness in the face of any concept it might encounter is proposed to give it the potential ability to solve any conceptual problem it might conceive, that is, general problem solving ability. In this way the FMF represents cognition as an adaptive process. This adaptive process is part of a hierarchy of adaptive processes which the FMF defines as being ordered by the sequence in which they evolved. Each adaptive process is defined by the FMF as providing the ability to adapt within a given domain by navigating a set of properties of that domain. For example, the first primitive organisms to arise on earth might have lacked the ability to adapt to any given property of their environment, perhaps being blind to gradients 
in substances useful or harmful to them. As a result they might not have been able to distinguish those properties in the environment, and their environment might then appear uniform in every direction.

As mentioned, representing living organisms as a hierarchy of domains of adaptability, the cognitive system is one domain of adaptation. From this perspective the development of the cognitive system provided the ability to adapt by defining and navigating a conceptual space representing the world around us. In the FMF the resolution of the conceptual space is proposed to be related to the maximum number of patterns that can be simultaneously detected as a single composite pattern, and therefore this resolution is related to the maximum complexity of reasoning that can be used to define a concept. As the resolution in conceptual space increased to enable more concepts defined by more complex reasoning, and therefore enabling more abstract concepts to be defined, the cognitive system's ability to conceptualize might allow events to take on abstract properties like time, or context, that might distinguish one event from another, creating the ability to locate their position in the cognitive space, and creating the ability to navigate an expanded region of cognitive space between those events, where that expanded region of cognitive space represents an expanded region of their environment. Such an ability to adapt to achieve stability in an expanded region of the environment would have enabled human beings to more reliably navigate that environment to find sparsely distributed resources of higher value that otherwise would not be reliably discoverable. If this pattern of new adaptive domains enabling navigation through a greater region of the environmental states space can be generalized, the next transformative evolution in mankind's capabilities can be expected to provide the ability to become aware of and to adapt over properties of the environment that were not previously accessible. Concrete examples might involve big data, new data models, or other properties that might not yet have been envisioned.

General Collective Intelligence (GCI) has been defined as a platform with the potential to combine groups of individuals into a single super-intelligence [2]. At some point in mankind's evolutionary history the theory of evolution assumes the existence of ancestors who lacked the capacity for abstract thought. The properties of GCI have been defined within the Functional Modeling Framework as the abstract thought enabling groups to coherently understand and reason as a single entity. In the same way that any random group of cells might exchange information, but we don't observe cells exchanging information in a coherent way that allows them to act as a system of cognition without that cognitive system having been specifically created by adaptive process such as evolution, groups orchestrating their decision-making in a coherent way that allows them to collectively reason as a single system of collective cognition might not be reliably achievable without that system of collective cognition being created in a way that enables them to do so. If according to the criteria above, the introduction of human intelligence to these ancestors might have been nature's most important innovation in the history of the earth to date by magnitude of impact because that introduction enabled accumulation of value at orders of magnitude higher levels, then if the properties of GCI as defined in the FMF enable adaptation and accumulation of resources within the collective cognitive domain, that could represent a similar opportunity for a multiple orders of magnitude increase in accumulation of value. This opportunity for adaptation across the entirely new domain of the collective cognition then suggests a similar potential change in impact.

The theory behind GCI suggests that entire classes of collective impact, including potentially the Sustainable Development Goals (SDGs) cannot reliably be achieved without GCI because of a whole host of reasons, including misalignment between these impacts and the interests of decision-makers, and because current non-GCI based decision-systems don't adapt in a way that reliably converges on addressing this misalignment, as well as other issues [2]. This theory also suggests that GCI allows groups to adapt in a way that vastly increases the collective capacity to solve the general problem of 
achieving collective well-being, increasing this capacity to the point that entire classes of collective impact may be reliably achievable [3]. One specific model of GCI defined within the FMF is the change engine [3]. Adaptability in the change engine is proposed to be achieved by maintaining a dynamical stability in collective well-being as the system of cognition navigates its environment (i.e. well-being changes but tends to be confined to a path that keeps it within a stable range). This adaptability to maintain well-being within a stable range within a given environment also defines a degree of sustainability within that environment (environmental sustainability). In economic terms the ability to create and sustain outcomes at whatever scale required translates to GCI enabling social, environmental, or other impacts to be self-funding at whatever scale required to serve the needs of the population.

Because of these properties, GCI has been suggested as being essential in addressing a number of the existential risks to human civilization that are related to capacity or lack of capacity for group decisionmaking to converge on optimal action. Case studies have been defined demonstrating how GCI could potentially increase impact on addressing environmental degradation until effects such as human-driven climate change might be reliably addressed at global scale [23]. Other case studies have been defined demonstrating how GCI might reliably end poverty [21], [24], or how GCI might reliably increase access to affordable healthcare, education, food security, housing, employment, and other resources so that universal access to those resources is sustainably and reliably achievable [3],[24].

In addition, GCI is based on a common model of cognition that being a functional model independent of implementation is also proposed to represent individual and artificial cognition. This model of cognition has been used to create a model for Artificial General Intelligence (AGI) [4]. AGI has been called "humanity's last invention" because of its potential to evolve itself as well as to potentially create other new technology faster than human beings might be able to [15]. The potential economic and other impacts of this capability have been suggested to be immense [5] - [10]. However, this potential impact is also a great risk [11]. Human civilizations without any adaptive systems to ensure a dynamic balance in collective fitness might be fundamentally unbalanced with respect to collective well-being, and to indicators of that well-being such as planetary tipping points. This is because the ability of intelligence to accumulate value at orders of magnitude greater levels removes the contraints on inequality. Without organic rather than imposed constraints on inequality that might simply be disobeyed, game theory modeling [25] suggests individuals might be forced to compete to harm collective well-being the most possible by unsustainably driving consumption to generate ever-increasing levels of inequality and waste. Or else those individuals might be replaced in their role by another party who will do the same in their place. On the other hand, human civilizations with such an adaptive system are proposed to have the capacity to reliably achieve balance with respect to those planetary tipping points. One way this is designed to be achieved in GCI as a specific example of such an adaptive system is by giving powerful competitive advantage to groups that cooperate to achieve collective well-being, or that cooperate to achieve impact on indicators of that well-being such as reducing consumption to sustainable levels or providing universal access to resources required for that well-being.

However, the patterns of cooperation required to reliably achieve competitive advantage for groups that cooperate to achieve mutual benefit may be complex as groups get larger and as the number of activities they might cooperate to achieve grows. Whether the participants were individuals, AGIs, or both, these processes might involve the design, manufacture, and every other part of the life-cycle of every product or service. For this reason the number of processes a group might utilize cooperation in, and the potential cooperative relationships between those processes, could be far larger than the capacity of any individual to manage. The intelligent cooperation within the model of GCI referred to in this paper suggests that by conveying a fitness advantage such patterns of cooperation might 
constrain the behavior of individual group members from non-cooperation while also incentivizing cooperation. Given that the complexity of tasks an AGI might be capable of might be much greater than the complexity an individual is capable of, the network of mutually beneficial cooperative relationships required to bind the collective well-being of mankind with the well-being of AGI in such a cooperating group would be too complicated to generate without automation. The specific type of automation required is part of GCI (the domain of adaptation through cooperation).

Without this network of cooperative relationships to create sufficient competitive advantage to reliably incentivize cooperation, and sufficient disadvantages to reliably discourage non-cooperation, then in order to be protected from the competition or non-cooperation of an AGI or it's owner, each individual would have to have all the resources required to provide these incentives or disincentives on their own. There is a saying that no single bucket of water can ever be worth all the water in the well. Because an AGI might by itself always have a problem solving advantage over any individual, and because more resources can always be gained by the AGI in taking over all resources of non-cooperating individuals than can possibly be provided by any individual as an incentive to cooperation or disincentive against non-cooperation of the AGI, then not only can AGI not be made safe, but AGI (whether acting on it's own or acting on behalf of a centralized owner) cannot be protected against. Having a potentially unbeatable competitive advantage, and having the ability to accumulate orders of magnitude greater levels of resource inequality, the cost of failing to protect against the competition or non-cooperation of an AGI is potentially all the economic value in existence that is not required to be invested in order to extract more value. Where GCI might be mankind's most valuable innovation, and the most valuable innovation after the creation of intelligence itself, AGI without GCI might be mankind's most costly one.

Other case studies suggest GCI may have the capacity to radically accelerate progress in all types of research by adding all human knowledge and reasoning into a single collective "conceptual space" (a single semantic model) that enables all theories to be tested against all observations of this existence. In such a scenario no theory would ever again be a "fringe" theory since all theories might be evaluated on their merits rather than on their following. GCI might also have the capacity to define and solve problems that are too complex for any individual human being to conceive [12], [13]. Where human beings are proposed to be limited to the problem definitions and solutions that can be resolved with concepts requiring detection of no more than $\mathrm{N}$ patterns in information simultaneously ( $\mathrm{N}$ degrees of separation in conceptual space), because collective processes with the capacity for resolving much higher levels of complexity might easily be defined, a GCI might be able to access problem definitions and solutions that can be resolved with concepts requiring $\mathrm{N}+\mathrm{M}$ degrees of separation with $\mathrm{M}>\mathrm{N}$. As likely is true with the human brain during much of its evolutionary history, at some limit in ability to detect Nth order patterns, and as a result at some limit in ability to perceive complexity, the gain in value might not be worth the cost. However, the far greater resources available to the collective could make much more ability to detect complexity worthwhile. Some application of this capacity include the ability to continually assess the validity of all theories in all areas, rather than being restricted to theories with a popular following. If, for example, humankind were presented with a book of scientific theories proven by a civilization a hundred million years more advanced than mankind, without collective intelligence to increase capacity for complexity human beings might lack the ability to assess the validity of those theories and might have a high probability of simply discarding them. This effect is commonly observed in groups, where group leaders who propose solutions that are too complex for the remainder of the group might not be able to be successful as leaders [20].

Assuming the claims that GCI might make collective impact such as the Sustainable Development Goals sustainably self-funding, and the claims that entire classes of collective impact can't reliably be 
achieved without GCI, are valid, then it might be true that the US\$23 trillion SDGs funding gap estimated by the United Nations can't reliably be bridged without GCI. Since this US\$23 trillion far exceeds the US\$500 billion budget for the largest project in human history (the US Interstate highway system), by economic impact this would potentially make GCI the most important innovation in the history of mankind.

GCI has other potential implications. Collective reasoning requires modeling knowledge and reasoning processes so they can be represented within a single collective conceptual space. Creating the ability of the GCI to reproduce, evolve, and otherwise adapt technology requires defining a "map" between representations in the collective cognition of all current designs of products or services, and the basic functional components from which all those designs might be composed. Defining functional models of all the products, services, and processes required to build a given product, and functional models of all the products, services, and processes required to build those components, all the way down to a basic set of functional elements that might be used to recreate all others, this map is proposed to have the potential to act as a collective genome that might provide a mechanism for recreating all technology after a global catastrophe. In addition, the representation of reasoning and understanding in terms of an individual or collective conceptual space is proposed to have the potential to create a languageindependence that might protect this and other knowledge from changes in human language, changes in storage format, or other changes that might impact the transmission of information. Efforts towards such storage and transmission of human civilization might benefit significantly from these aspects of GCI [14]

Another potential implication is that GCI gives the collective the capacity to adapt to achieve dynamical stability within a given environment, that is, GCI has the potential to give civilizations environmental sustainability. A GCI is designed to achieve this ability to adapt in a stable way over the possible states of the environment through the domains of adaptability defined within the Functional Modeling Framework (FMF) that apply to both individuals and collectives. In order for this collective adaptability over each of these domains to be dynamically stable so that it is sustainable, this adaptability must be governed by stability in collective fitness to continue to function. In groups of human-beings this collective fitness in the FMF is associated with the well-being of the collective consciousness. This need for dynamical stability in collective well-being in order to achieve collective sustainability in a given environment raises an important point. A GCI is a first order collective intelligence in an Nth order pattern. A collective intelligence consisting of collective intelligences is defined in the FMF as second order, and so forth. If a first order GCI must have a collective well-being defined for it to have the ability to adapt in a stable way within a given environment, the limits to that stability, and hence the limits to that environmental sustainability, would logically only extend to the point at which populations don't share a common definition of well-being that applies across shared environmental states. For example, a first order GCI might give permit human civilization to harvest and accumulate the resources of entire solar systems. However, if these resources are required for other planetary civilizations to have the ability to escape consequences we might later learn all planetary civilizations might otherwise be likely to fall prey to, then this ability might lead to depleting resources in a way that is unstable in the well-being of other planetary civilizations and therefore environmentally unsustainable in that larger sense. In order to achieve stability between multiple such civilizations a second order GCI might be required. That second order GCI will have its own limits to stability that might require a third order GCI, and so forth.

As a competitive advantage for it's owner, AGI has the potential to be a massive generator of inequality, accumulating wealth from any markets in which transactions can be triggered through electronic communication [5] - [10]. And through doing so for the owner of the most powerful AGI 
potentially at a pace orders of magnitude greater than for others [16] - [18]. Without GCI all decisionmaking systems are proposed to lack the decentralization required to ensure outcomes are optimized for all participants [3]. The resulting unbalanced centralization is proposed to align decision-making with the interests of the subset of decision-makers with the greatest influence over decisions. The difference between the outcomes that can be achieved and the optimal outcomes possible with GCI as a decentralized system of decision-making is one cost of centralization of authority. This cost is proposed to in essence be a "tax" on all transactions within societies governed by a subset of powerful decisionmakers, even those transactions not directly involving them. Game theory modeling suggest this tax steadily accumulates benefit to them. Increases in inequality, the increases in technological capacity for surveillance and control, both of which will certainly be provided at unprecedented levels by an AGI, all would increase this tax due to the influence resources have on decision-making in a centralized system. Taxes aren't generally limited by the capacity of a people to bear them, but by the capacity of the tax to be imposed. The most extreme examples of cruelty and other abuse of authority in human history likely arose with levels of authority and immunity against consequences that were far less than what might be possible with AGI.

Finally, despite life being priceless to some, putting an economic value on a human life is routine in some industries such as insurance in which that economic value used to quantify the benefit of avoiding a fatality. In many studies the value also includes the quality of life, the expected life time remaining, as well as the earning potential of a given person especially for an after the fact payment in transactions requiring that information, such as a wrongful death claim lawsuit. The value of a human life is relevant to environmental sustainability because there might be essentially only two paths to achieving sustainability. One is through a decentralized system of decision-making such as GCI that maximizes collective well-being per unit of resources, the other is continuing along the path of centralization towards an authoritarian system of decision-making. Assuming that centralized systems of decisionmaking lack the capacity to reliably maximize collective outcomes [3] that leaves only the option of simply reducing the number of people and therefore the demand for resources. Not implementing GCI might mean implicitly depending on the latter. Of course, population reduction can occur through voluntary incentives to reduce reproduction or to reduce lifespan, or through involuntary coercion to do the same. In the sense that there is an economic value to human life, whether that of the individual or their families or others they might have invested in, there is a cost associated with such population reduction. There is also a cost (the "tax" imposed by alignment with the interests of decision-makers) associated with the level of authoritarianism required to implement such control over individual liberties. Because of that tax, in an authoritarian system the cost might then tend to be be distributed among the majority, while the value might then tend to be accumulated by decision-makers.

Observation of authoritarian rule confirms that authoritarian rulers generally do not share any economic suffering of the populace they rule over. Given that, by the arguments in this paper, AGI without GCI might strongly tend to support the creation of such authoritarianism, these are some of the additional costs that might be anticipated with AGI implemented without GCI.

\section{References}

[1] Where is the Wealth of Nations?: Measuring Capital for the 21st Century, World Bank e-Library Editor Kirk Hamilton, World Bank (2006), ISBN 0821363549,9780821363546

[2] Andy E. Williams, A Model for General Collective Intelligence, under review

[3] Andy E. Williams, The Need for General Collective Intelligence in Addressing the Sustainable Development Goals, under review

[4] Andy E. Williams, A Model for Artificial General Intelligence, under review

[5] Bundy, A. Preparing for the future of Artificial Intelligence. AI \& Soc 32, 285-287 (2017).

https://doi.org/10.1007/s00146-016-0685-0 
[6] Ajay Agrawal, Joshua Gans, and Avi Goldfarb, Economic Policy for Artificial Intelligence, Innovation Policy and the Economy 2019 19:, 139-159

[7] Gans, J. (2017). Self-regulating artificial general intelligence. Retrieved March 24, 2020, from https://www.nber.org/papers/w24352 . [Google Scholar]

[8] Ramamoorthy, Anand, and Roman Yampolskiy. 2018. Beyond Mad? The Race for Artificial General Intelligence. ITU Journal: ICT Discoveries 1: 1-8. [Google Scholar]

[9] Baum, Seth, A Survey of Artificial General Intelligence Projects for Ethics, Risk, and Policy (November 12, 2017). Global Catastrophic Risk Institute Working Paper 17-1 . Available at SSRN: https://ssrn.com/abstract=3070741. or http://dx.doi.org/10.2139/ssrn.3070741

[10] Ted Goertzel (2014) The path to more general artificial intelligence, Journal of Experimental \& Theoretical Artificial Intelligence, 26:3, 343-354, DOI: 10.1080/0952813X.2014.895106

[11] Vincent C. Müller (2014) Risks of general artificial intelligence, Journal of Experimental \& Theoretical Artificial Intelligence, 26:3, 297-301, DOI: 10.1080/0952813X.2014.895110

[12] Funke, J. (1991). Solving complex problems: Exploration and control of complex systems. In R.J. Sternberg \& P.A. Frensch (eds.), Complex problem solving: Principles and mechanisms (pp. 185-222). Hillsdale, NJ: Lawrence Erlbaum Associates.

[13] N. Johnson, S. Rasmussen, C. Joslyn, L. Rocha, S. Smith, M. Kantor, "Symbiotic intelligence: self-organizing knowledge on distributed networks driven by human interaction", 6th International Conference on Artificial Life, 1998.

[14] Clara Moskowitz, DNA-Coded "Lunar Library" Aims to Preserve Civilization for Millennia, Published September 28, 2018, Retrieved March 24, 2020, from

https://www.scientificamerican.com/article/dna-coded-lunar-library-aims-to-preserve-civilization-formillennia/

[15] Divya S Ganji, Sharayu Karandikar, Artificial Intelligence: A Risk Factor, International Research Journal of Engineering and Technology (IRJET), Volume: 06 Issue: 04 | Apr 2019

[16] Unver, Hamid Akin, Artificial Intelligence, Authoritarianism and the Future of Political Systems (July 15, 2018). EDAM Research Reports, 2018. Available at SSRN:

https://ssrn.com/abstract $=3331635$

[17] Feldstein, Steven. (2019). "How Artificial Intelligence Systems Could Threaten Democracy". The Conversation

[18] Nicholas Wright, "How Artificial Intelligence Will Reshape the Global Order," Foreign Affairs, 10 July 2018, https://www.foreignaffairs.com/articles/world/2018-07-10/how-artificial-intelligence-willreshape-global-order

[19] Andy E. Williams, A Functional Modeling Framework for Representing and Comparing Models of Consciousness or Cognition, under review

[20] Antonakis, J., House, R. J., \& Simonton, D. K. (2017). Can super smart leaders suffer from too much of a good thing? The curvilinear effect of intelligence on perceived leadership behavior. Journal of Applied Psychology, 102(7), 1003-1021. https://doi.org/10.1037/ap10000221

[21] Andy E. Williams, The Design of the Social Impact Marketplace as a Partial Implementation of a General Collective Intelligence Platform, working paper (2020)

[22] Andy E. Williams, Conceptual Healthcare Platforms as Partial Implementations of a General

Collective Intelligence Platform, working paper (2020)

[23] Andy E. Williams, A Proposed Collective Intelligence Based Renewable Energy Solution

Development Program, working paper (2020)

[24] Andy E. Williams, The Collective Intelligence based Program to Accelerate Achievement of the

Sustainable Development Goals (CIPAA-SDGs) as a Case Study for Collectively Intelligent Program Design, working paper (2019)

[25] Andy E. Williams, The Principles Enabling Cooperation within Conscious Organisms and

Collectives, working paper (2019) 